\title{
SFTA2 - A NOVEL PULMONARY SURFACTANT PROTEIN SECRETED BY TYPE II CELLS - IS DOWNREGULATED IN LPS-INDUCED LUNG INFLAMMATION
}

\author{
M. Hammel ${ }^{1}$, R. Mittal ${ }^{1}$, J. Schwarz ${ }^{2}$, K.M. Heschl ${ }^{1}$, S. Herber-Jonat ${ }^{1}$, O. Eickelberg ${ }^{2}$, A. Holzinger ${ }^{1}$ \\ ${ }^{I}$ Neonatology, Ludwig-Maximilians-Universität, ${ }^{2}$ CPC Großhadern, Comprehensive Pulmonology Center, \\ München, Germany
}

Tissue-specific transcripts are likely to play a significant role in the function of the corresponding organ. In an in silico approach to define the specific transcriptome of the human lung, we identified the transcript of a yet uncharacterized protein, SFTA2. The human SFTA2 gene is located on Chr 6p21.33, a diseasesusceptibility locus for diffuse panbronchiolitis also encoding mucin21 and mucin-like genes. Specific transcription of the SFTA2 gene in the human lung was verified by RT-PCR. SFTA2 encodes a small hydrophilic precursor peptide ( 78 amino acids) releasing a 59 amino acid mature peptide after cleavage of an amino-terminal secretory signal. Human SFTA2 has no recognizable homology to other human proteins while orthologues are present in all mammals. Specific antibodies raised against the human peptide recognized a $13 \mathrm{kDa}$ Peptide in human respiratory epithelial cells with a shift to the expected $6 \mathrm{kDa}$ after deglycosylation. In immunofluorescence staining of human lung sections, signals corresponding to SFTA2 were specifically detected in Type II pneumycytes of the alveolar epithelium. While SFTA2 showed no colocalization with lamellar bodies, it was detected in human broncho-alveolar lavage fluid thus establishing this peptide as a novel hydrophilic surfactant protein. In the mouse, Sfta2 was significantly downregulated after induction of a pulmonary inflammatory reaction by intratracheal administration of lipopolysaccharides indicating regulated secretion. We speculate about a possible role of SFTA2 in innate immune defence and inflammation. 